\title{
Mutations and thrombosis in essential thrombocythemia
}

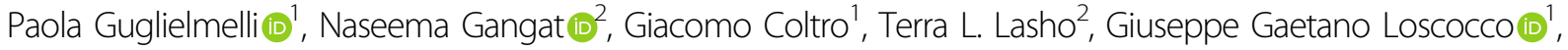 \\ Christy M. Finke², Erika Morsia², Benedetta Sordi', Natasha Szuber², Curtis A. Hanson? ${ }^{3}$, Animesh Pardanani², \\ Alessandro M. Vannucchi ${ }^{1}$ and Ayalew Tefferi ${ }^{2}{ }^{2}$
}

Dear Editor,

Essential thrombocythemia (ET) constitutes one of the three JAK2/MPL/CALR-mutated myeloproliferative neoplasms (MPNs), which also include polycythemia vera (PV) and primary myelofibrosis (PMF) ${ }^{1}$. ET is defined by clonal thrombocytosis (platelet count $\geq 450 \times 10^{9} / \mathrm{L}$ ) and characteristic bone marrow megakaryocyte morphology; the clinical phenotype in ET might include leukocytosis, splenomegaly, microvascular symptoms, and thrombohemorrhagic complications ${ }^{1}$. Most patients with ET enjoy a near-normal life expectancy while disease progression into fibrotic or leukemic transformation is relatively infrequent $(<1 \% \text { in the first } 10 \text { years of disease })^{2}$. Collaborative studies between the Mayo Clinic, Rochester, MN, USA and University of Florence, Florence, Italy have recently established an integrated clinical and genetic survival risk model for $\mathrm{ET}^{3}$. According to this new mutation-enhanced international prognostic model for ET (MIPSS-ET), independent risk factors for overall survival included SF3B1/SRSF2 mutations (occurring in $10 \%$ of patients), age $>60$ years, male sex, and leukocytosis $\left(\geq 11 \times 10^{9} / \mathrm{L}\right)$. To date, drug therapy has not been shown to influence survival in ET and is instead directed at decreasing the risk of thrombosis. In the latter regard, four risk categories are recognized: very low risk (age $\leq 60$ years, no thrombosis history, JAK2 wild type), low risk (age $\leq 60$ years, no thrombosis history, JAK2 mutated), intermediate risk (age $>60$ years, no thrombosis history, $J A K 2$ wild type) and high risk (thrombosis history or age

\footnotetext{
Correspondence: Ayalew Tefferi (tefferi.ayalew@mayo.edu)

'Department of Experimental and Clinical Medicine, CRIMM, Center of Research and Innovation of Myeloproliferative Neoplasms, Azienda

Ospedaliera Universitaria Careggi, University of Florence, Florence, Italy

2Divisions of Hematology, Mayo Clinic, Rochester, MN, USA

Full list of author information is available at the end of the article
}

$>60$ years with $J A K 2$ mutation) ${ }^{1}$. In the current two-center study involving 502 patients (seen between 1967 through 2017) with World Health Organization (WHO)-defined ET, we sought to further clarify the influence of driver and other mutations on both arterial and venous thrombosis, in the context of other established risk factors.

Study patients were recruited from the Mayo Clinic, Rochester, MN, USA and the University of Florence, Florence, Italy, based on the availability of nextgeneration sequencing (NGS)-derived mutation information (Table 1). Diagnosis of ET was based on the 2016 WHO criteria ${ }^{4}$. Methods for mutation screening have previously been published; a detailed account of all mutations investigated including their variant allele frequency has previously been published ${ }^{3,5}$. Conventional statistics was employed to examine the significance of associations, with separate analysis of arterial vs venous thrombosis, occurring at any time before or after formal diagnosis of ET. For the purposes of the current study, only the first events were considered.

A total of 502 patients (median age 55 years; $41 \%$ above age 60 ; females $59 \%$ ) who were molecularly annotated for driver mutations as well as other mutations derived from NGS data were followed for a median of 10 years (0.1-34.7). The Mayo/Florence cohorts included 270/232 patients (median age 57/54 years, 60\%/59\% females) with median follow-up of 9.9/12.9 years (Table 1). Overall driver mutation distribution was 55\% JAK2, 27\% CALR, 5\% $M P L$, and $13 \%$ triple-negative (TN); among 132 CALRmutated cases, 78 (59\%) harbored type-1/like and 54 (41\%) type-2/like $C A L R$ mutations ${ }^{6}$. Most frequent mutations, other than JAK2/CALR/MPL, were ASXL1 (7-20\%), TET2 (9-11\%), DNMT3A (7\%), SF3B1 (5\%), SRSF2 (2-3\%), EZH2 (2-4\%), TP53 (2-4\%), RUNX1 (1-2\%), CBL (1-2\%), $I D H 2(1 \%)$ and $U 2 A F 1(1 \%)$. Leukocytosis $\left(\geq 11 \times 10^{9} / \mathrm{L}\right)$ 
Table 1 Clinical and laboratory characteristics of $\mathbf{2 7 0}$ Mayo Clinic patients and 232 University of Florence patients with essential thrombocythemia (ET) (total $n=502)$.

\begin{tabular}{|c|c|c|}
\hline Variables & $\begin{array}{l}\text { Mayo Clinic } \\
(n=270)\end{array}$ & $\begin{array}{l}\text { Florence } \\
(n=232)\end{array}$ \\
\hline $\begin{array}{l}\text { Age in years; median } \\
\text { (range) }\end{array}$ & $57(18-92)$ & $54(13-85)$ \\
\hline Males; $n$ (\%) & $108(40)$ & $96(41)$ \\
\hline $\begin{array}{l}\text { Hemoglobin, g/dl; median } \\
\text { (range) } \\
\text { "N" evaluable = } 382(92 \%)\end{array}$ & $13.7(6.9-17.9)^{\mathrm{a}}$ & $14.1(12-17.0)^{\mathrm{a}}$ \\
\hline $\begin{array}{l}\text { Platelets, } \times 10^{9} / L \text {; median } \\
\text { (range) } \\
\text { " } \mathrm{N} \text { " evaluable }=407(98 \%)\end{array}$ & $844(451-3330)$ & $739(451-2000)$ \\
\hline $\begin{array}{l}\text { Platelets }>1000 \times 10^{9} / \mathrm{l} \\
n(\%) \\
" N " \text { evaluable }=407(98 \%)\end{array}$ & $82(31)$ & $33(16)$ \\
\hline $\begin{array}{l}\text { Leukocytes, } \times 10^{9} / \mathrm{L} \text {; median } \\
\text { (range) } \\
\text { "N" evaluable }=399(96 \%)\end{array}$ & $8.7(2.7-70.7)$ & $8.5(3.8-26)$ \\
\hline $\begin{array}{l}\text { Leukocytes } \geq 11 \times 10^{9} / \mathrm{l} ; \\
n(\%) \\
\text { "N" evaluable = } 399(96 \%)\end{array}$ & $64(25)$ & $36(19)$ \\
\hline $\begin{array}{l}\text { Palpable splenomegaly } \\
\text { "N" evaluable = } 412 \text { (99\%) }\end{array}$ & $48(18)$ & $45(21)$ \\
\hline \multicolumn{3}{|c|}{ Karyotype "N" evaluable = 345 (83\%) } \\
\hline Abnormal; $n$ (\%) & $20(9)$ & $15(10)$ \\
\hline Fibrotic progression; $n(\%)$ & $44(16)$ & $76(33)^{b}$ \\
\hline $\begin{array}{l}\text { Leukemic transformations; } \\
n(\%)\end{array}$ & $12(4)$ & $15(6.5)$ \\
\hline $\begin{array}{l}\text { Follow up in years; } \\
\text { median (range) }\end{array}$ & $9.9(0-34.6)$ & $12.9(1-36.3)$ \\
\hline Deaths; $n(\%)$ & $104(39)$ & $87(38)$ \\
\hline \multicolumn{3}{|l|}{ Mutations $^{c}$} \\
\hline JAK2 mutated; $n(\%)$ & $146(54)$ & $129(56)$ \\
\hline CALR mutated; $n$ (\%) & $79(29)$ & $59(25)$ \\
\hline TET2 mutated; $n(\%)$ & $25(9)$ & $28(11)$ \\
\hline ASXL1 mutated; $n(\%)$ & $18(7)$ & $47(20)^{b}$ \\
\hline DNMT3A mutated; $n(\%)$ & $19(7)$ & $14(7)$ \\
\hline SF3B1 mutated; $n(\%)$ & $14(5)$ & $12(5)$ \\
\hline SH2B3 mutated; $n(\%)$ & $3(1)$ & $6(3)$ \\
\hline SRSF2 mutated; $n(\%)$ & $6(2)$ & $6(3)$ \\
\hline MPL mutated; $n(\%)$ & $11(4)$ & $17(7)$ \\
\hline KIT mutated; $n$ (\%) & $5(2)$ & $2(1)$ \\
\hline
\end{tabular}

Table 1 continued

\begin{tabular}{lcc}
\hline Variables & $\begin{array}{l}\text { Mayo Clinic } \\
(\boldsymbol{n}=\mathbf{2 7 0})\end{array}$ & $\begin{array}{l}\text { Florence } \\
(\boldsymbol{n}=\mathbf{2 3 2})\end{array}$ \\
\hline IDH2 mutated; $n$ (\%) & $4(1)$ & $2(1)$ \\
TP53 mutated; $n$ (\%) & $5(2)$ & $9(4)$ \\
U2AF1 mutated; $n$ (\%) & $3(1)$ & $6(2.5)$ \\
RUNX1 mutated; $n$ (\%) & $4(1)$ & $5(2)$ \\
EZH2 mutated; $n(\%)$ & $5(2)$ & $9(4)$ \\
CBL mutated; $n$ (\%) & $3(1)$ & $3(2)$ \\
\hline
\end{tabular}

aET patients who had low hemoglobin, presented with concomitant bleeding disorders, iron deficiency anemia, chronic renal insufficiency, or other rare disorders such as sickle cell anemia and Osler-Weber-Rendu disease.

${ }^{\mathrm{b}}$ The difference in the frequency of ASXL1 mutation and fibrotic transformation in the two patient cohorts is explained by the intentional enrichment of the Florence cohort with patients who had transformed to myelofibrosis for the purposes of a prior project examining the predictive value of mutations for postET fibrotic transformation.

Included mutations with frequency of at least $1 \%$. Also, the denominator for the percentages in parenthesis is the number of evaluable cases.

was documented in $22 \%$ of patients, extreme thrombocytosis $\left(\geq 1000 \times 10^{9} / \mathrm{L}\right)$ in $27 \%$ and abnormal karyotype in $9 \%$. Patients were managed according to conventional strategies including cytoreductive drugs for high-risk disease and antiplatelet therapy for low-risk disease.

History of thrombosis (arterial or venous) before or after diagnosis was documented in 152 (30\%) patients and included arterial events in $96(19 \%)$ and venous in $82(16 \%)$. The number of arterial/venous vascular events before and after the time of formal diagnosis were $50(10 \%) / 3(1 \%)$ and $87(17 \%) / 76(15 \%)$. In univariate logistic regression analysis the incidence of all thrombotic events (i.e. arterial or venous) occurring before or after diagnosis was significantly associated with driver mutation profile $(p<0.01$; JAK2 36\%, CALR 20\%, MPL 26\%, and TN 32\%) and the absence of ASXL1 $(p=0.04)$ or IDH mutations $(p=0.01)$. A similar analysis restricted to arterial events occurring before or after diagnosis revealed a near-significant association for driver mutation profile $(p=0.2$; JAK2 $22 \%$, CALR 14\%, MPL 22\%, and TN 19\%) and significant associations for the absence of ASXL1 $(p=0.02)$ or RUNX1 $(p=0.05)$ mutations; a similar analysis for venous events marked driver mutation profile ( $p=0.03$; JAK2 21\%, CALR $10 \%, M P L 15 \%$ and TN $13 \%)$ and absence of SRSF2 ( $p=$ $0.03)$ or $E Z H 2(p=0.02)$ mutations as being significant. In a subsequent multivariable logistic regression analysis that included age $>60$ years and leukocytosis $\left(\geq 11 \times 10^{9} / \mathrm{L}\right)$, significance was confirmed for JAK2 vs CALR $(p<0.01)$, absence of $I D H(0.01)$ or ASXL1 ( $p=0.06)$ mutations, for all thrombosis; JAK2 vs CALR $(p<0.01)$ and absence of SRSF2 $(p=0.02)$ or EZH2 $(p=0.03)$ mutations, for venous thrombosis; and absence of ASXL1 $(p=0.02)$ or RUNX1 $(p=0.05)$ mutations for arterial events; of note, in all 


\section{Thrombosis risk associations in essential thrombocythemia (ET)}

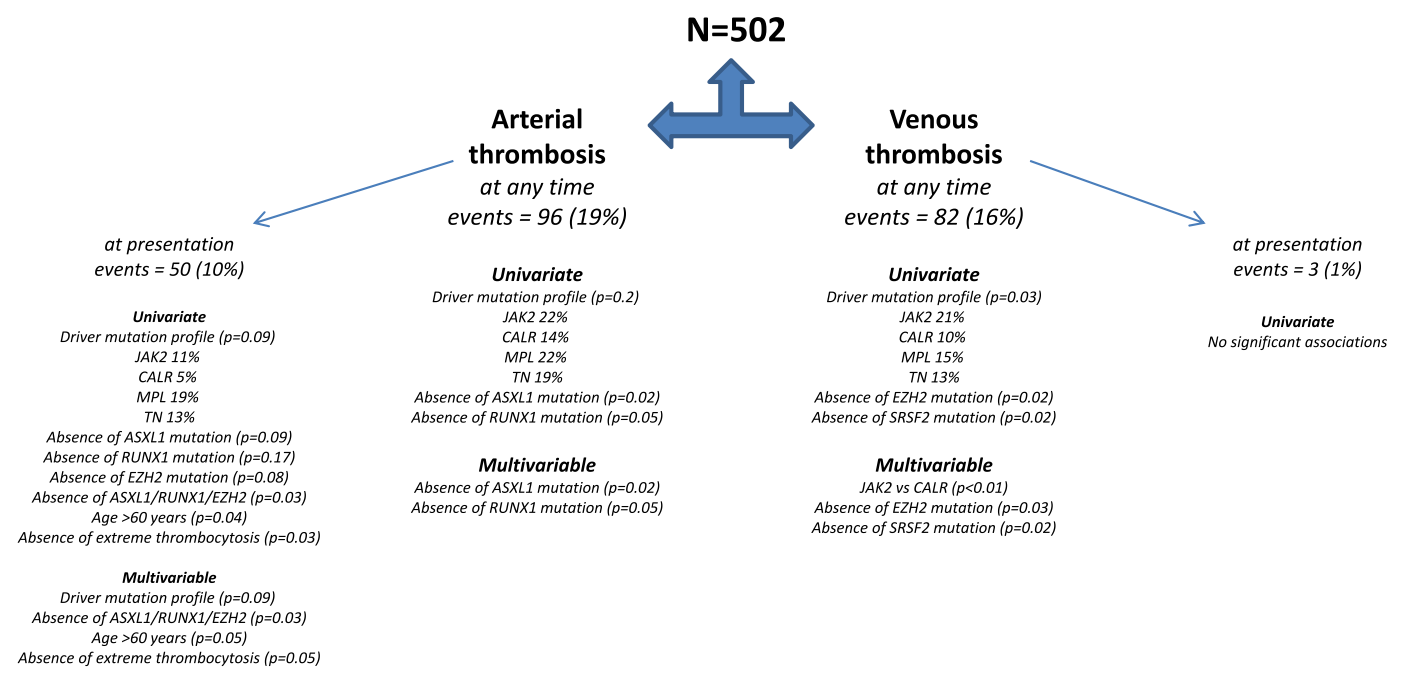

Conclusions:

1. ASXL1, RUNX1 or EZH2 mutations in ET were associated with decreased risk of arterial thrombosis, which suggests a different underlying biology

2. Extreme thrombocytosis, often seen in CALR-mutated ET, might partly explain the latter's association with decreased risk of arterial thrombosis

Fig. 1 Thrombosis risk associations in essential thrombocythemia.

instances, significant associations were not apparent for either leukocytosis or age $>60$ years.

Next, in order to mitigate the confounding effect of treatment, we queried for associations with thrombotic events occurring at or before the time of diagnosis; during such analysis, extreme thrombocytosis $\left(\geq 1000 \times 10^{9} / \mathrm{L}\right)$ was also included as variable of interest, based on previous reports of its association with lower risk of arterial thrombosis $^{7}$ as well as $C A L R$ mutations ${ }^{8}$. In univariate analysis, the following were found to be significantly or nearsignificantly associated with an increased risk of arterial events: absence of extreme thrombocytosis $(p=0.03 ; 12 \%$ vs $5 \%)$, age $>60$ years $(p=0.04)$, absence of $A S X L 1 \quad(p=$ $0.09), E Z H 2(p=0.08), R U N X 1(p=0.17)$ mutations, wildtype ASXL1/RUNX1/EZH2 genotype ( $p=0.03$; $9 \%$ vs $1 \%$ ), and driver mutation profile $(p=0.09$; JAK2 11\%, CALR 5\%, MPL 19\%, and TN 13\%); leukocytosis was not significant ( $p$ $=0.8$ ); multivariable analysis confirmed significance of wild-type ASXL1/RUNX1/EZH2 genotype $(p=0.03)$, age $>60$ years $(p=0.05)$ and absence of extreme thrombocytosis $(p=0.05)$, while driver mutation profile was relegated to borderline significance $(p=0.09)$. A similar analysis for venous events did not reveal any significant association. Finally, for arterial vascular events occurring after diagnosis (i.e. in the context of ongoing anti-thrombotic therapy), logistic regression analysis identified $J A K 2$ vs $C A L R$ $(p<0.01)$ and wild-type ASXL1/RUNX1/EZH2 genotype $(p=0.07)$ as being significant or near-significant. Cox progression analysis for arterial thrombosis-free survival was feasible in the Mayo Clinic cohort and confirmed the significant unfavorable effect of wild-type ASXL1/RUNX1/ $E Z H 2$ genotype $(p=0.02)$, and also identified a previous arterial event as an independent risk factor $(p=0.04)$.

Figure 1 summarizes our findings in the current study. Our main observations included (i) salutary effect of ASXL1/RUNX1/EZH2 mutations on the risk of arterial thrombosis in ET and (ii) prognostic interaction between extreme thrombocytosis and CALR mutation in influencing the incidence of arterial events at the time of diagnosis. The favorable influence of harboring ASXL1/ RUNX1/EZH2 mutations on arterial thrombosis was evident in the context of arterial events occurring both before and after diagnosis. To that effect, it is reasonable to consider the possibility that the infrequent occurrence of high-risk mutations might be a marker for a biologically different disease phenotype, such as occult prefibrotic myelofibrosis ${ }^{9}$. In a previous report in ET, overall survival was adversely affected by spliceosome (SF3B1, SRSF2) and leukemia-free survival by TP53 mutations ${ }^{3}$. In a more recent report of young patients with ET, extreme 
thrombocytosis was independently associated with shortened overall and leukemia-free survival whereas there was no such influence from driver mutations including $J A K 2$ or $C A L R^{10}$.

Extreme thrombocytosis in ET has previously been associated with both lower risk of arterial thrombosis ${ }^{7}$ and CALR mutations ${ }^{8}$. CALR mutations in ET have also been associated with decreased risk of thrombosis, which in the past has been attributed to the younger age distribution of affected cases ${ }^{11,12}$. The current study confirms the prothrombotic influence of $J A K 2$, as opposed to $C A L R$ mutation ${ }^{13}$, and suggests that extreme thrombocytosis might also play a part in contributing to the observed decreased risk of arterial thrombosis in CALRmutated ET. Additional studies are required to confirm these observations, assess their clinical impact, and provide additional insight into the underlying biological mechanisms, which are likely to include the longrecognized, platelet count-dependent, development of acquired von Willebrand syndrome ${ }^{14}$.

\section{Author details}

${ }^{1}$ Department of Experimental and Clinical Medicine, CRIMM, Center of Research and Innovation of Myeloproliferative Neoplasms, Azienda Ospedaliera Universitaria Careggi, University of Florence, Florence, Italy. ${ }^{2}$ Divisions of Hematology, Mayo Clinic, Rochester, MN, USA. ${ }^{3}$ Divisions of Hematopathology, Departments of Internal Medicine and Laboratory Medicine, Mayo Clinic, Rochester, MN, USA

\section{Conflict of interest}

The authors declare no competing interests.

\section{Publisher's note}

Springer Nature remains neutral with regard to jurisdictional claims in published maps and institutional affiliations.
Received: 10 January 2021 Revised: 4 March 2021 Accepted: 7 April 2021 Published online: 27 April 2021

\section{References}

1. Tefferi, A. \& Barbui, T. Polycythemia vera and essential thrombocythemia: 2021 update on diagnosis, risk-stratification and management. Am. J. Hematol. 95, 1599-1613 (2020).

2. Szuber, N. et al. Myeloproliferative neoplasms in the young: Mayo Clinic experience with 361 patients age 40 years or younger. Am. J. Hematol. 93, 1474-1484 (2018)

3. Tefferi, A. et al. Mutation-enhanced international prognostic systems for essential thrombocythaemia and polycythaemia vera. Br. J. Haematol. 189, 291-302 (2020).

4. Arber, D. A. et al. The 2016 revision to the World Health Organization classification of myeloid neoplasms and acute leukemia. Blood 127, 2391-2405 (2016).

5. Tefferi, A. et al. Targeted deep sequencing in polycythemia vera and essential thrombocythemia. Blood Adv. 1, 21-30 (2016).

6. Lasho, T. L., Finke, C. M., Tischer, A., Pardanani, A. \& Tefferi, A. Mayo CALR mutation type classification guide using alpha helix propensity. Am. J. Hematol. 93, E128-E129 (2018).

7. Carobbio, A. et al. Risk factors for arterial and venous thrombosis in $\mathrm{WHO}$ defined essential thrombocythemia: an international study of 891 patients. Blood 117, 5857-5859 (2011).

8. Tefferi, A. et al. Type 1 versus Type 2 calreticulin mutations in essential thrombocythemia: a collaborative study of 1027 patients. Am. J. Hematol. 89, E121-E124 (2014).

9. Barbui, T. et al. Survival and disease progression in essential thrombocythemia are significantly influenced by accurate morphologic diagnosis: an international study. J. Clin. Oncol. 29, 3179-3184 (2011).

10. Gangat, N. et al. Young platelet millionaires with essential thrombocythemia Am. J. Hematol. https://doi.org/10.1002/ajh.26114 (2021).

11. Finazzi, G. et al. Calreticulin mutation does not modify the IPSET score for predicting the risk of thrombosis among 1150 patients with essential thrombocythemia. Blood 124, 2611-2612 (2014).

12. Gangat, N. et al. Mutations and thrombosis in essential thrombocythemia: prognostic interaction with age and thrombosis history. Eur. J. Haematol. 94, 31-36 (2015).

13. Barbui, T. Refining prognostication of thrombosis in ET. Am. J. Hematol. 91, 361-363 (2016).

14. Michiels, J. J. et al. The paradox of platelet activation and impaired function: platelet-von Willebrand factor interactions, and the etiology of thrombotic and hemorrhagic manifestations in essential thrombocythemia and polycythemia vera. Semin. Thromb. Hemost. 32, 589-604 (2006). 